\title{
Laboratory diagnosis of pneumonia in the molecular age
}

\author{
Antoni Torres ${ }^{1}$, Nelson Lee ${ }^{2}$, Catia Cilloniz ${ }^{1}$, Jordi Vila ${ }^{3}$ and Menno Van der Eerden ${ }^{4}$
}

\author{
Affiliations: \\ ${ }^{1}$ Dept of Pneumology, Institut Clinic del Tórax, Hospital Clinic of Barcelona, Institut d'Investigacions \\ Biomèdiques August Pi i Sunyer (IDIBAPS), University of Barcelona, Ciber de Enfermedades Respiratorias \\ (CIBERES), Barcelona, Spain. \\ ${ }^{2}$ Division of Infectious Diseases, Dept of Medicine and Therapeutics, Faculty of Medicine, The Chinese University \\ of Hong Kong, Hong Kong, China. \\ ${ }^{3}$ Servei de Microbiologia, Hospital Clinic, ISGlobal, Barcelona Ctr. Int. Health Res. (CRESIB), School of Medicine, \\ Universitat de Barcelona, Barcelona, Spain. \\ ${ }^{4}$ Dept of Respiratory Medicine, Erasmus Medical Centre, Rotterdam, The Netherlands.
}

\section{Correspondence:}

Antoni Torres, Dept of Pneumology, Hospital Clinic of Barcelona, C/ Villarroel 170, 08036 Barcelona, Spain.

E-mail: atorresaclinic.ub.es

ABSTRACT Pneumonia remains a worldwide health problem with a high rate of morbidity and mortality. Identification of microbial pathogens which cause pneumonia is an important area for optimum clinical management of pneumonia patients and is a big challenge for conventional microbiological methods. The development and implementation of molecular diagnostic tests for pneumonia has been a major advance in the microbiological diagnosis of respiratory pathogens in recent years. However, with new knowledge regarding the microbiome, together with the recognition that the lungs are a dynamic microbiological ecosystem, our current concept of pneumonia is not totally realistic as this new concept of pneumonia involves a dysbiosis or alteration of the lung microbiome. A new challenge for microbiologists and clinicians has therefore arisen. There is much to learn regarding the information provided by this new diagnostic technology, which will lead to improvements in the time to antibiotic therapy, targeted antibiotic selection and more effective de-escalation and improved stewardship for pneumonia patients. This article provides an overview of current methods of laboratory diagnosis of pneumonia in the molecular age.

@ERSpublications

Integration of novel diagnostics for rapid microbial identification will impact laboratory diagnosis of pneumonia http://ow.ly/2aJh304NuCn 


\section{Introduction}

In 1930, pneumonia was the third most frequent cause of death in the USA [1]. Now, almost a century later, and despite the introduction of antibiotics in the 1950s, pneumonia and other respiratory infections remain the fourth most common cause of death worldwide [2]. Although the implementation of vaccination programmes throughout the world has reduced the incidence of pneumonia, especially in at-risk populations $[3,4]$, pneumonia still represents a major burden in terms of morbidity, mortality and health costs, as well as days of work lost [5-8].

Microbiological diagnosis of pneumonia is fundamental to ensure appropriate antibiotic therapy, which is associated with decreasing mortality [9]. However, aetiological diagnosis is only achieved in half of patients and the initial antibiotic regimen should be chosen empirically in order to avoid the delay in instating appropriate therapy that is associated with significant mortality $[10,11]$.

The Infectious Disease Society of America/American Thoracic Society (IDSA/ATS) guidelines for community-acquired pneumonia (CAP) recommend an optional microbiological diagnostic test in low-level to mild cases of pneumonia, which should be selected as clinically indicated [12]. However, routine microbiology should be performed in the case of pathogens that would significantly alter empirical decisions, such as the influenza virus, Mycobacterium tuberculosis, Pseudomonas aeruginosa and especially methicillin-resistant Staphylococcus aureus (MRSA), because the current guideline recommendation for severe CAP is empirical therapy with $\beta$-lactam with macrolide or fluoroquinolone, which may not provide adequate protection against MRSA [12]. Sputum cultures are only recommended for patients ill enough to require intensive care unit (ICU) admission. This means that, depending on the epidemiology of microbial aetiology, empirical treatments are chosen and given to patients as soon as possible after culture sample collection.

In current practice, most CAP patients can be treated following guidelines. The use of scores can help to detect those patients with potential risk for multidrug-resistant pathogens or patients who require non-standard treatment. [13-15] In these patients, molecular information, in addition to conventional techniques, may be very useful. Current clinical practices have three main consequences. 1) The adequacy of the initial antibiotic is the key factor for prognosis. It is known that mortality increases with each hour of delay in initiating the correct therapy for pneumonia [16-19]. 2) Many patients receive broad-spectrum antibiotic therapies until cultures are available and de-escalation cannot be performed quickly; inappropriate use of antibiotics is thus more likely [20, 21]. 3) The emergence of antibiotic-resistant pathogens related to selection pressure by over use of antibiotics worldwide [22, 23].

Antimicrobial resistance among Gram-positive cocci and Gram-negative bacilli is a major problem worldwide [18, 23, 24]. The chapter in the State of the World's Antibiotics report 2015 [25] on the human use of antibiotics reports that consumption continues to increase (comparative data 2000-2010), and that antibiotic treatment is inappropriate in between 20 and $50 \%$ of cases. The data on consumption per capita by class and country reveal considerable variability between geographical locations, and this underlines how much countries' responses to the problem can vary [25]. Public campaigns encouraging the implementation of specific measures have been successful $[26,27]$. Most campaigns of this kind include calls and warnings for better and more rapid diagnostic testing in order to reduce antibiotic administration and to increase the use of microbe targeted therapies. The problem of microbial resistance is so serious that, last year, President Obama drew attention to the threat it poses and called for greater research funding [28]. However, the discovery of novel classes of antibiotics is more or less at a standstill, as the costly and inefficient process is a challenge for pharmaceutical companies, who have abandoned their investment in developing new antimicrobials. The Generating Antibiotic Incentives Now (GAIN) is a new regulation of the Food and Drug Administration (FDA) aimed at providing economic incentives for antibiotic drug discovery by offering market exclusivity and accelerated drug approval processes. The regulation extends the exclusivity with which antibiotics that treat serious or life-threatening illnesses can be sold without generic competition by 5 years. It also provides an additional 6 months of exclusivity to the pharmaceutical or biotechnological company that identifies a companion diagnostic test. Additionally, the FDA grants the option of accelerating the approval for the new antimicrobial [29].

Rapid molecular techniques (figure 1 and table 1) promise to be an effective tool to help guide appropriate therapy and de-escalation from broad-spectrum antibiotic therapy. However, like any new medical technology, they need to be validated in clinical practice.

In this article, we assess the most recent information on non-molecular and molecular methods for detecting respiratory pathogens such as bacteria and viruses. The conclusions of this review are that for most of the pathogens that cause pneumonia, there is the possibility of using rapid methods that may help clinicians to use a better-targeted antibiotic and antiviral treatment. Point-of-care testing will be the best way to implement these methods in clinical practice. However, before implementing these methods, well-designed studies must be performed to determine their cost-effectiveness. 


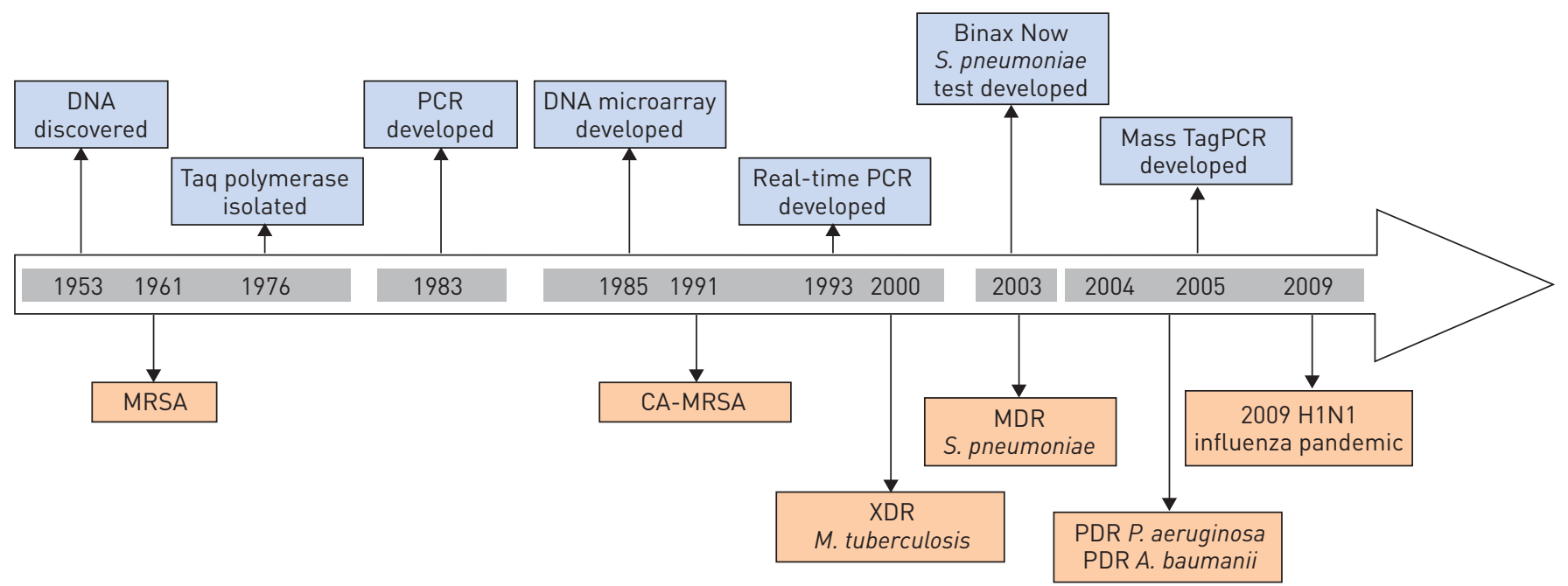

FIGURE 1 Timeline for diagnostic test development and emergence of resistant pathogens. MRSA: methicillin-resistant Staphylococcus aureus; CA-MRSA: community-acquired methicillin-resistant Staphylococcus aureus; MDR: multidrug-resistant: PDR: pan drug-resistant; XDR: extensively drug-resistant.

\section{Diagnosis of lower respiratory tract infections caused by conventional bacteria Conventional microbiological diagnosis}

In patients with CAP, microbiological investigation should consist of at least a sputum culture, a urinary antigen test for Streptococcus pneumoniae and Legionella pneumophila, and a blood culture [12]. Additional serological investigation for atypical bacterial pathogens may be performed [12]. In studies involving intensive microbiological investigation in patients with CAP, an aetiology was identified in 53-75\% of the sample [30-32]. The most common pathogens identified in these studies were S. pneumoniae, Mycoplasma pneumoniae and Haemophilus influenzae.

Blood cultures performed before administration of antibiotic therapy have a very high specificity but are positive in less than $20 \%$ of cases [12,33]. Blood cultures from patients with severe CAP have a higher yield because pathogens such as $S$. aureus and Gram-negative bacilli are frequently isolated and are not affected by empirical therapy [12].

Pleural effusion is present in approximately $40 \%$ of CAP cases [34]. The specificity of pleural exudate culture is very high, although the sensitivity is low because of the low incidence of invasion of the pleura [34]. The sensitivity of the Gram stain is approximately $80 \%$ in cases of pneumococcal pneumonia [35] and $78 \%$ for staphylococcal pneumonia, with a specificity of $93-96 \%$ [36].

Detection of S. pneumoniae using PCR

PCR is a molecular diagnostic technique based on DNA detection and offers the advantage of providing results within a few hours. Furthermore, as PCR does not require viable bacteria, it is less influenced by antimicrobial therapy. In a study by JOHANSSON et al. [37], in 184 consecutively admitted patients with diagnosis of CAP, $80 \%$ of cases with a positive PCR test and negative sputum culture had been treated with antibiotics prior to sputum sampling. In another study on the effect of antibiotic treatment on the accuracy of diagnosing invasive pneumococcal disease using culture or molecular methods, an aetiological diagnosis was established using molecular methods after 4 days or more of antibiotic treatment, and only in the first 2 days of treatment using conventional diagnostic methods [38].

PCR-based detection of $S$. pneumoniae depends on the amplification of pneumococcus-specific genes. Specific characteristics for this species are the production of toxins such as pneumolysin $(P l y)$, as well as the presence of surface antigens such as pneumococcal surface adhesin A (PsaA) and pneumococcal autolysin A ( LytA) [39]. However, studies investigating PCRs directed at the ply gene showed a lack of sensitivity and specificity for pneumococcal disease [40]. It has also been shown that the ply gene was present in some species of Streptococcal mouth flora such as Streptococcus mitis and Streptococcus oralis [41]. To investigate whether gene targets other than the ply gene might be more specific, CARVALHO et al. [41] compared two other targets: the lytA gene and the $p s a A$ gene. They showed that a PCR designed to target the lyt $A$ gene was $100 \%$ specific, while that for the $p s a A$ gene was $98 \%$ specific. Another study confirmed the high specificity for the lytA gene, in which no positive results were found in healthy control subjects [52]. 
TABLE 1 Rapid microbiological tests for pneumonia diagnostics

Platform

Pathogens

Technology

Sensibility/

specificity \%

\section{Clinical evidence}

Time/cost

Sample

Curetis Unyvero system (P50 pneumonia) [42]

\section{Bacterial/fungal pathogens Multiplex-PCR (cartridge (18 types) \\ system}

resistance markers

(22 types)

MRSA

Multiplex-PCR

Gene Xpert MRSA/SA $[43,44]$

Microorganisms (200 types)

S Identification directly from bacterial/fungal

colonies

Gene Xpert Flu Assay [48]

Influenza A/B (A/2009 H1)

Multiplex-PCR

Multiplex-PCR

Influenza A/B/RSV

Gene Xpert Flu/RSV Assay [48]

eSensor Respiratory

Viral Panel [49]

\section{FilmArray Respiratory}

Panel $[50,51]$
Influenza A/B Iseasonal H1, Multiplex-PCR

$$
\begin{aligned}
& \text { H3, } 2009 \text { H1) } \\
& \text { RSV A/B }
\end{aligned}
$$

Parainfluenza 1, 2,

Human metapneumovirus

$$
\text { Rhinovirus }
$$

Adenovirus B/E/C

$$
\text { Adenovirus }
$$

Nucleic acid purification, Coronavirus 229E, OC43, NL63, HKU1

Metapneumovirus

Influenza A, H3, H1, 2009 H1

Parainfluenza virus $1,2,3,4$. RSV high-order nested multiplex-PCR and DNA melting curve analysis (unprocessed biological/ clinical sample needed)

Rhinovirus/enterovirus Bordetella pertussis

$$
\text { M. pneumoniae }
$$

C. pneumoniae
$81 / 99$

Potential for accurate and timely detection of pathogens and their resistance in severe pneumonia

99-100/97-100

Rapid, accurate tool for detecting MRSA and MSSA in blood and

respiratory samples

Rapid identification of

microorganisms in BAL was

associated with adjustment of antibiotic therapy and a shorter ICU

stay for ventilated patients with

$$
\text { pneumonia }
$$

Detects certain types of antibiotic resistance mechanisms

Rapid identification of

influenza virus in outbreaks

$97-100 / 100$

Rapid identification of influenza virus in outbreaks

$98-99 / 99$

Rapid identification of respiratory viruses

Co-infection detection

84-100/98-100

Detection of several respiratory pathogens in one test

$1 \mathrm{~h} / € 100-120$

Nasopharyngeal swabs

$4 \mathrm{~h} / € 280-300 \quad$ Sputum

$1 \mathrm{~h} / € 40$

BAS

$\cos 2$

$\begin{array}{cc}30 \mathrm{~s}-1 \mathrm{~min} / & \text { Colonies } \\ € 0.50-1.00 & \text { Positive blood cultures }\end{array}$ Direct samples

(e.g. urine)

Significant impact on the care of

patients with respiratory infections

MALDI-TOF: matrix assisted laser desorption/ionisation time-of-flight; MS: mass spectrometry; MRSA: methicillin-resistant S. aureus; MSSA: methicillin-sensitive S. aureus; RSV: respiratory syncytial virus; BAL: bronchoalveolar lavage; BAS: bronchial aspirate; ICU: intensive care unit 
The diagnosis of pneumococcus using PCR tests can be applied to respiratory samples, nasopharyngeal swabs and blood.

\section{Sputum: PCR versus culture}

Several studies have investigated the ability of PCR to detect $S$. pneumoniae in sputum $[37,53-55]$. In two Scandinavian studies, sputum was collected to perform PCR and culture. In the first study, 128 and 127 patients with CAP had at least one sputum sample analysed by culture and real-time PCR, respectively; the PCR targeted the pneumolysin (ply) gene of S. pneumoniae [37]. Sputum PCR was positive in 34 patients $(27 \%)$ while sputum culture was positive in 19 patients $(15 \%)(p<0.001)$. Sixteen of the 19 patients with a positive culture (84\%) also had positive results with real-time PCR and 17 of the 34 patients with positive real-time PCR (50\%) had negative cultures. Fourteen of these 17 patients (82\%) had been treated with antibiotics before sputum sampling. In the second study, a sputum culture was performed in 112 patients and PCR in 103 patients: 36 patients (32\%) had positive cultures and 55 patients (53\%) had positive PCR results [56]. These studies suggest that sputum PCR is a more sensitive method than sputum culture for detecting $S$. pneumoniae in patients hospitalised with CAP, especially in those previously treated with antibiotics.

Blood/plasma: PCR versus culture

Blood cultures have a low sensitivity for detecting S. pneumoniae infections [30]; bacteraemia is found to be present in about $20 \%$ of CAP cases where conventional blood cultures are used [33]. PCR may be able to increase this sensitivity. One study, which assessed the role of PCR in whole blood when targeted at the ply gene, showed a positive result in 22 of 40 patients (55\%) with pneumococcal pneumonia, while blood culture had a sensitivity of $28 \%$ (11 of 40 patients). The PCR was negative in all 30 patients with non-pneumococcal pneumonia, giving a specificity of 100\% [57]. Two studies in adult patients determined the sensitivity of positive PCR results compared to positive blood cultures as a gold standard. In the first study, 10 CAP patients had pneumococcal bacteraemia, PCR for lytA had a sensitivity of $70 \%$ (seven out of 10) [58]. The second study showed a positive PCR lytA result for 10 out of 13 patients (77\%) with S. pneumoniae bacteraemia [59]. Furthermore, in a review assessing the value of PCR for diagnosing pneumococcal bacteraemia, a sensitivity of $57.1 \%$ was reported (95\% CI $45.7-67.8 \%$ ). The PCR specificity of $98.6 \%$ (95\% CI 96.4-99.5\%) was determined in a population of "patients with no disease", defined as healthy people or patients with bacteraemia caused by other bacteria [60].

Other PCR studies targeted at the lytA gene showed better performance with PCR than with blood culture. In a prospective study including children younger than 15 years of age and adults with CAP, significantly more cases of definite pneumococcal pneumonia were detected by PCR than by blood culture $(27.1 \%$ versus $5.0 \%$; $\mathrm{p}<0.005)$. PCR in plasma was negative in a control group of 50 adults [52]. In another study, including children aged 0-16 years, invasive pneumococcal disease was diagnosed in 47 of 292 patients (16\%). Of these patients, $45(15.4 \%)$ tested positive with PCR and $11(3.8 \%)$ tested positive with culture. PCR was thus significantly more sensitive than culture in revealing bacteraemic pneumonia (OR 30.6, 95\% CI 5.8-97.5; $\mathrm{p}<0.001$ ) [61]. The results of these two studies showed that molecular methods had higher sensitivity than blood cultures in bacteraemic patients. One of the explanations may be that PCR can also detect DNA from non-viable bacteria. In the latter case, the sensitivity of blood cultures may be influenced by the fact that antibiotic treatment was started prior to culture collection. In one study, in patients with CAP who received antibiotic treatment before hospital admission, PCR sensitivity was 7.0 times higher than that of culture $(\mathrm{p}=0.043)$ [38].

Once a positive result is obtained from a PCR test in respiratory samples it is important to determine whether this result reflects bacterial colonisation or infection. Recently, quantitative PCR assays have been performed in different clinical samples (whole blood, sputum and nasopharyngeal specimens) that may differentiate between colonisation and infection. In these studies, a cut-off of $10^{4}$ to $10^{5}$ colony forming units per $\mathrm{mL}$ of pneumococcal DNA was described in non-HIV patients $[58,62,63]$. Furthermore, there is some evidence that a quantitative approach can also be used to predict disease presentation and severity, which may correlate with levels of pneumococci DNA in clinical PCR samples. In a prospective study of 353 patients with a diagnosis of CAP, ReLlo et al. [64] showed that the median number of copies per $\mathrm{mL}$ of $S$. pneumoniae DNA in whole blood samples was significantly higher in patients in whom septic shock developed. In two other studies, a higher S. pneumoniae DNA load in blood samples was also associated with the presence of more severe disease [59, 65]. Another beneficial application of PCR is that this diagnostic method can be used for pneumococcal serotyping in cases of bacteraemia [61, 66]. This is important for the identification of serotypes that may be responsible for severe CAP cases. These new tests may also be useful for identifying the circulating pneumococcal serotypes in order to assess the effect of pneumococcal vaccination. 
Additionally, PCR has the potential to recognise genes inducing antibiotic resistance [67]. At present, however, conventional cultures remain the gold standard for determining resistance to antibiotics.

\section{Diagnosis of viral and atypical pneumonia}

Viral and atypical pathogens are common causes of pneumonia worldwide, representing $10-22 \%$ and $11-28 \%$ of all CAP cases, respectively [68-72]. In a recent study by JAIN et al. [68] analysing 2320 cases of pneumonia where an intensive microbiological diagnosis was applied, particularly viral molecular techniques, microbial aetiology was identified in 853 (38\%) cases. The three main causal agents found were respiratory viruses $(23 \%)$, bacterial aetiology (11\%) and co-infections (3\%). This study was particularly important in putting two issues into context. 1) The isolation of viruses in CAP is much more frequent than was previously thought, thanks to molecular techniques; and 2) Although molecular techniques are better than microbiological cultures, these methods are not perfect in terms of operational values.

Diagnosis commonly involves antigen tests, culture, serology and molecular assays, all of which have their own advantages and limitations (table 2), such as: specimen type, site and time of collection with respect to illness onset, and the pretest probability (e.g. seasonal peaks), which may significantly affect test performance [73-75]. Testing is generally recommended in hospitalised patients because specific antimicrobial treatments (e.g. neuraminidase inhibitors for influenza, or macrolides, fluoroquinolones, or doxycycline for atypical pathogens) are available and diagnosis may assist effective implementation of isolation precautions $[70,73,74,76,77]$.

Influenza (seasonal, pandemic, avian) is the most important cause of viral pneumonia, leading to high morbidity and mortality; however, clinical diagnosis is unreliable [73, 74]. Rapid Influenza Diagnostic Tests (RIDT, antigen immunoassays) are convenient, commercially available and highly specific (90-95\%). The major drawback is their low sensitivity $(40-70 \%)$, which means that a negative result cannot rule out influenza infection [74, 78-80]. The tests' performance does improve if they are applied within 48-72 h of illness onset, before significant viral-load drop occurs [74, 75]. Similar considerations apply to immunofluorescence antigen assays, although they are generally more sensitive (50-85\%), can be used in upper and lower respiratory specimens (e.g. tracheal aspirates, bronchoalveolar lavage) and can detect a range of respiratory viruses (e.g. respiratory syncytial virus (RSV), parainfluenza virus) if included in the panel. Laboratory expertise is essential, as is the availability of quality specimens with abundant epithelial cells for staining [73, 74]. Generally, serology and culture cannot assist patient care because of their retrospective or slow results, but they are useful for surveillance and research purposes (e.g. for strain identification and phenotypic resistance testing) [74, 81, 82].

Where available, PCR is now considered the test of choice, due to its high sensitivity and specificity, greater time window for detection and rapid turnaround time [73, 74, 80]. It can detect all influenza A subtypes with the universal primers (targeting the M-gene, as for influenza B), or individual subtypes using specific primers (e.g. H1, H3, H5, H7) [83]. This information may have implications for treatment (e.g. pre-pandemic $\mathrm{H} 1 \mathrm{~N} 1$ is oseltamivir-resistant) and may identify possible novel or avian strains (e.g. H1N1pdm09 and H7N9 are initially "untypable" using existing primers) [78, 81, 83, 84].

Even with PCR, negative results may occur with nasopharyngeal samples in the case of influenza pneumonia due to differential viral kinetic changes along the respiratory tract. Additional testing with a lower respiratory sample (in which viral loads are generally higher) should therefore be considered $[75,78,84,85]$.

Notably, PCR does not provide information regarding infectiousness and dead virus RNA fragments may be detected, albeit at a low level, even upon clinical resolution [83]. Semi-quantitative, real-time assays may be useful for the monitoring of virologic response (cycle-threshold values have been used as alternative estimates) [75, 82, 85]. Rapid, direct detection of genotypic resistance is also possible with PCR for known mutations (e.g. H275Y in H1N1pdm09) [81-83].

As manifestations of influenza are indistinguishable from other respiratory pathogens, multiplex PCR platforms that detect a range of common viruses (e.g. influenza, RSV, human metapneumovirus, parainfluenza virus, rhinovirus) and atypical pathogens (e.g. M. pneumoniae, Chlamydophila pneumoniae) are increasingly being used in the clinical setting [68, 72, 80, 86]. More recently, molecular-based point-of-care tests (loop-mediated isothermal amplification (LAMP) of nucleic acids, real-time PCR) have become available for detecting influenza and other viruses at the bedside, offering a degree of accuracy comparable to conventional laboratory PCR assays [77, 80]. However, the impact of these molecular assays on clinical outcomes and their cost-effectiveness remain unclear and they deserve further evaluation [87].

RSV causes major morbidity in children and older adults, with burdens, manifestations and severity comparable to influenza. Specific antivirals (e.g. fusion inhibitors) are in the pipeline [88, 89]; however, diagnosis can be difficult because of the low sensitivity of antigen assays (20-60\%, higher in children), and culture is slow and 


\section{Pathogen Diagnostic method}

\begin{tabular}{|c|c|c|c|c|}
\hline & Culture & Antigen test & Blood culture & PCR \\
\hline S. pneumoniae & $\begin{array}{l}\text { Positive in } 60-80 \% \text { of good quality } \\
\text { sputum samples } \\
\text { Between } 17 \text { and } 35 \% \text { of CAP patients } \\
\text { can expectorate sputum of } \\
\text { adequate quality }[3,52,60]\end{array}$ & $\begin{array}{l}\text { Results in }<30 \mathrm{~min} \\
\text { ICT: sensitivity } 60-85 \% \text { and specificity } 99 \% \\
\text { UAD: sensitivity } 98 \% \text { and specificity } 99 \% \text { [60] }\end{array}$ & $\begin{array}{l}\text { Definitive diagnosis } \\
\text { Positive in less than } 10 \% \text { of } \\
\text { pneumococcal CAP cases } \\
\text { Results in } 1-2 \text { days }[52,60]\end{array}$ & $\begin{array}{l}\text { Results within hours } \\
\text { High sensitivity/specificity } \\
\text { Allows diagnosis of early, low bacterial } \\
\text { load disease } \\
\text { Lower influence of previous antibiotic } \\
\text { treatment } \\
\text { Accessibility, technical demands and } \\
\text { cost are practical concerns, as is lack } \\
\text { of standardisation }[3,60,71]\end{array}$ \\
\hline \multirow[t]{2}{*}{ L. pneumophila } & $\begin{array}{l}\text { Sensitivity } 50-80 \% \text { with high } \\
\text { specificity ("gold standard") } \\
\text { Allows detection of different } \\
\text { L. pneumophila strains } \\
\text { Technically demanding-requires } \\
\text { optimal sampling and } \\
\text { post-sampling processing } \\
\text { conditions } \\
\text { False-negative results may occur in } \\
\text { late or treated infections } \\
\text { Results are too slow to guide patient } \\
\text { care [9] }\end{array}$ & $\begin{array}{l}\text { Results in }<30 \text { min } \\
\text { Sensitivity } 75-80 \% \text {; specificity } 99-100 \% \\
\text { Unreliable for non-serogroup } 1 \text { infections } \\
\text { Positive results may indicate recent infection } \\
\text { "False-negatives" may occur in early infection } \\
\text { Concomitant culture of respiratory secretions in } \\
\text { selective media is recommended, especially } \\
\text { in immunocompromised patients }[90,91]\end{array}$ & & $\begin{array}{l}\text { Results within hours } \\
\text { High sensitivity and specificity } \\
\text { Allows diagnosis of early, low bacterial } \\
\text { load, and non-serogroup } 1 \text { infections } \\
\text { Accessibility, technical demands and } \\
\text { cost are practical concerns } \\
\text { Lack of standardisation } \\
\text { Respiratory specimens may be } \\
\text { unavailable [57] }\end{array}$ \\
\hline & RIDT [92] & DFA or IFA $[83,84,92]$ & Viral cell culture $[84,89,93]$ & RT-PCR $[76,80,94,95]$ \\
\hline Influenza virus & $\begin{array}{l}\text { Immunoassay for antigens } \\
\text { Results in }<30 \text { min } \\
\text { Applicable to a range of upper } \\
\text { respiratory samples } \\
\text { Differentiates between influenza A/B } \\
\text { Specificity } 90-95 \% \\
\text { Sensitivity } 40-70 \% \\
\text { Unable to distinguish between } \\
\text { virus subtypes }\end{array}$ & $\begin{array}{l}\text { Direct or indirect immunofluorescence antibody } \\
\text { staining } \\
\text { Results within hours } \\
\text { Applicable to upper and lower respiratory } \\
\text { samples } \\
\text { Detects influenza A, B and other viruses } \\
\text { simultaneously if included in the panel } \\
\text { Specificity } 90-95 \% \\
\text { Sensitivity } 50-85 \% \\
\text { A quality specimen containing adequate } \\
\text { epithelial cells together with laboratory } \\
\text { expertise is essential } \\
\text { Unable to distinguish between virus subtypes }\end{array}$ & $\begin{array}{l}\text { Conventional or shell-vial viral } \\
\text { cell culture } \\
\text { High specificity } \\
\text { Sensitivity } 7-20 \% \\
\text { Allows virus subtyping, strain } \\
\text { identification, titre assay/ } \\
\text { quantification and resistance } \\
\text { testing } \\
\text { Provides information on } \\
\text { infectiousness } \\
\text { Requires optimal sampling and } \\
\text { post-sampling processing } \\
\text { conditions } \\
\text { Results too slow to guide patient } \\
\text { care (conventional culture: } \\
\text { >3-10 days; shell-vial culture: } \\
\text { >2-3 days) }\end{array}$ & $\begin{array}{l}\text { Reverse-transcription PCR } \\
\text { Results within hours } \\
\text { High sensitivity and specificity ("gold } \\
\text { standard") } \\
\text { Applicable to a wider range of specimen } \\
\text { types } \\
\text { Able to distinguish between virus } \\
\text { subtypes and detect genotypic } \\
\text { resistance } \\
\text { Multiplex PCR methods may allow } \\
\text { simultaneous detection of other } \\
\text { respiratory pathogens } \\
\text { Accessibility, technical demands and } \\
\text { cost are practical concerns } \\
\text { Unable to distinguish non-viable from } \\
\text { viable viruses } \\
\text { "False-negatives" may result from } \\
\text { site-differential viral kinetic changes } \\
\text { in pneumonia cases }\end{array}$ \\
\hline
\end{tabular}




\section{TABLE 2 Continued}

\section{Pathogen Diagnostic method}

\section{Serology $[96,97]$}

\section{Molecular assay [97]}

Results within hours

High sensitivity, moderate-to-high specificity

Accessibility, technical demands and cost are practical concerns

Lack of standardisation

Specimen of choice is uncertain

Cannot distinguish acute infection from asymptomatic carriage, especially in children

Paired acute and convalescent serum (2-4 weeks apart) are required

Results are thus retrospective

"False-positives" may occur with IgM tests and with previous infection

CAP: community-acquired pneumonia; ICT: immunochromatographic assay; UAD: serotype-specific urinary antigen detection assay; RIDT: rapid influenza diagnostic test; DFA: direct immunofluorescence antibody staining; IFA: indirect immunofluorescence antibody staining; ELISA: enzyme-linked immunosorbent assay; CFT: complement fixation test. 
technically challenging $[87,88]$. The more sensitive PCR assays (singleplex, multiplex) are becoming the tests of choice, especially in adults who generally present with lower viral loads [80, 87, 94]. Similarly, in RSV pneumonia, lower respiratory samples including sputum should also be considered if available; use of a quality sputum sample and methods to overcome the difficulties in its processing have been suggested [72, 85, 87]. Testing for other viruses (commonly with multiplex assays) is gaining importance because of the increasing recognition of their roles in causing lower respiratory infections, hospitalisations and ICU admissions; clinical trials of broad-spectrum or virus-specific agents are underway (e.g. nitazoxanide-NCT01227421, favipiravir-NCT01728753 and DAS181-NCT01644877) [68, 72, 77, 83, 88]. Lessons from epidemics caused by emerging pathogens suggest that careful laboratory evaluation of undiagnosed pneumonia with molecular (e.g. H1N1pdm09, H7N9) and culture (e.g. severe acute respiratory syndrome coronavirus, Middle East respiratory syndrome coronavirus) methods, together with application of advanced techniques such as next-generation sequencing, are important for their prompt detection [78, 88, 92].

Traditionally, atypical bacterial pathogens are not routinely diagnosed due to difficulties in performing cultures and the lack of reliable tests $[69,70]$. Single-titre IgM assays provide quicker results but are limited by their moderate sensitivity (e.g. early disease, attenuated IgM response in adults) and specificity (e.g. past infection, other infectious/non-infectious diseases) [95, 98].

Recent studies indicate high sensitivity for rapid molecular tests (e.g. LAMP, singleplex PCR, multiplex PCR) which can be applied to a range of respiratory specimens including sputum and bronchoalveolar lavage $[80,98]$. Nevertheless, the methods have not been standardised (e.g. primer targets) and the optimal specimen types remain uncertain. Improvements in diagnosis are urgently needed to guide specific therapy because of the emergence of macrolide resistance worldwide (Asia 30-100\%, USA/Europe 10-30\%) and the challenge this poses for empirical regimens against CAP (typically a $\beta$-lactam plus a macrolide) [95]. Similar issues exist for the serological and molecular diagnosis of acute C. pneumoniae infection, including prolonged asymptomatic carriage and lack of standardisation [93]. In contrast, diagnostic methods for L. pneumophila infection are well established and are described in current pneumonia management guidelines [93, 99]. Briefly, urine antigen tests have high sensitivity (75-80\%) and specificity (99-100\%), but only reliably diagnose the commonest serogroup (serogroup 1). More recent data suggest that PCR of lower respiratory specimens, including sputum, allows the diagnosis of early, low bacterial load and non-serogroup 1 infections. However, the presence of a dry cough may limit its use. Combining PCR with urine tests may maximise the diagnostic yield $[68,93,100]$.

\section{Diagnosis of pneumonia caused by potentially multidrug-resistant bacteria}

The most frequent multidrug-resistant bacteria involved in pneumonia are MRSA, P. aeruginosa, Acinetobacter baumannii and various Enterobacteriaceae [18, 23, 101-103]. The reference diagnostic techniques used to identify the bacteria causing respiratory tract infections remain the Gram stain and semi-quantitative conventional culture from direct respiratory samples, followed by bacterial identification using MALDI-TOF (matrix-assisted laser desorption/ionisation time-of-flight) mass spectrometry [48, 104, 105] and susceptibility testing of the potential pathogen [106]. In addition to the difficulty of differentiating between colonisation and infection, this process requires a minimum of 2 days and has low sensitivity, especially if the sample is taken after the start of antibiotic treatment [39]. As a consequence, many patients receive inappropriate antibiotic treatment, which may increase morbidity and mortality $[16,96,97,107]$. This statement is also valid for conventional bacteria.

Current guideline recommendations for multidrug-resistant pathogens (MRSA, P. aeruginosa and drug-resistant $S$. pneumoniae) include the use of respiratory fluoroquinolone or ß-lactam plus macrolide in outpatients. For inpatients, ICU patients and suspected $P$. aeruginosa infections, the use of an anti-pneumococcal, anti-pseudomonal ß-lactam plus either ciprofloxacin or levofloxacin; ß-lactam plus an aminoglycoside; or B-lactam plus an aminoglycoside and an anti-pneumococcal fluoroquinolone are recommended. For community-acquired MRSA, the recommendation is to add vancomycin or linezolid.

There are several scoring systems to identify CAP patients with risk factors for acquiring multidrugresistant pathogens. Of these, two systems, the clinical scores by SHOrR et al. [14] and AliberTi et al. [13] categorise risk factors into groups: In the system devised by SHORR et al. [14], points are allocated for recent hospitalisation (4 points), nursing home residence ( 3 points), haemodialysis ( 2 points) and ICU admission ( 1 point). Patients with less than 3 points have a $20 \%$ prevalence of multidrug-resistant pathogens whereas patients with more than 5 points have a prevalence of 75\%. The system developed by ALIBERTI et al. [13] assigns points to hospitalised patients with CAP involving chronic renal failure (5 points), prior hospitalisation ( 4 points), residence in a nursing home ( 3 points) and other risk factors ( 0.5 points each). Patients with at least 3 points have a prevalence of multidrug-resistant pathogens of $38 \%$ whereas patients with a score of $\leqslant 0.5$ points have a prevalence of $8 \%$. Molecular diagnosis, such as with dedicated PCR assays, has partly overcome the difficulties mentioned [90]. In recent years, molecular techniques based on 
multiplex PCR have also been developed in order to simultaneously identify and quantify multiple respiratory pathogens from different types of samples in a single procedure (table 3) [44, 49-51, 91].

Another challenge for the rapid diagnosis of respiratory infections is early detection of the antibiotic resistance profile of the various bacteria [42]. The biggest obstacle in the use of molecular techniques for detecting resistance is the discrepancy between genotype and phenotype [108]. New resistance mechanisms are continually being discovered and, as a result, the potential presence of unknown mechanisms may lead to false-negative results using molecular techniques [109]. An additional problem is the detection of genotypic markers which phenotypically do not show clinically significant resistance [108].

Despite the drawbacks of molecular techniques, early information on the resistance profile is more likely to improve empirical treatment than no information at all [109]. An assay based on multiplex PCR is currently under development, which can detect some bacterial resistance markers involved in causing respiratory infection, including the genes related to $\beta$-lactam resistance (mecA, bla $a_{\mathrm{TEM}}, b l a_{\mathrm{SHV}}, b l a_{\mathrm{CTX}-\mathrm{M}}$, $b l a_{\mathrm{DHA}}, b l a_{\mathrm{EBC}}, b l a_{\mathrm{OXA}-51}$ and $\left.b l a_{\mathrm{KPC}}\right)$, macrolides/lincosamides (ermA, ermB, ermC, msrA and mefA), fluoroquinolones (detection of changes in the amino acid codons gyrA83and gyrA87 of GyrA and in ParC) and markers of class 1 integrons (int1 and sul1) [110].

Recently, a new group of molecular techniques has emerged based on LAMP [45]. Unlike conventional PCRs, in which a series of temperature change cycles are required for the amplification of nucleic acids, these techniques allow amplification at a constant temperature. ZHANG et al. [46] applied this technique to the rapid diagnosis of major pathogenic bacteria (S. pneumoniae, S. aureus, Escherichia coli, Klebsiella pneumoniae, P. aeruginosa, A. baumannii, Stenotrophomonas maltophilia and H. influenzae) in 120 sputum samples from patients with suspected chronic obstructive pulmonary disease exacerbations. LAMP obtained more positive results than conventional cultures and a higher correlation with clinical symptoms as well.

In a recent study by KONCAN et al. [47], a new methodology called "respiFISH" was developed that combined classical fluorescence in situ hybridisation (FISH) technology with fluorescence-labelled DNA molecular beacons as probes. This was used to analyse 165 respiratory samples, including 85 sputa, 79 bronchial secretions (75 bronchial aspirates and four bronchial lavages) and one tracheal aspirate. The sensitivity of this methodology was $94.39 \%$ and its specificity was $87.93 \%$. The hands-on time of the assay was approximately $30 \mathrm{~min}$.

VINCENT et al. [112] described a PCR followed by electrospray ionisation mass spectrometry, which could detect more than 800 infection-relevant bloodstream pathogens in a single assay in patients with sepsis and or

TABLE 3 Laboratory diagnosis of pneumonia caused by potentially multidrug-resistant bacteria

$\begin{array}{lll}\text { Diagnostic method Advantages Disadvantages } & \text { Adval }\end{array}$

Culture $[3,46,69]$

Molecular assay (Singleplex)

$[3,57,61,69,75,78]$

\section{Molecular assay (Multiplex)} $[9,43,65,105,111]$
Easy to perform and cheap

Isolation and identification of bacteria whose detection by other methods has not been standardised

(i.e. S. maltophilia)

The susceptibility of the isolated pathogens can be assessed

High turnaround time

High sensitivity and specificity

Allows diagnosis of early, low bacterial load disease

Lower influence of antibiotic pretreatment compared to conventional culture

Allows detection of resistance markers (i.e. mec gene for MRSA)

High turnaround time (1-6 h)

High sensitivity and specificity

Allows diagnosis of early, low bacterial load disease

Lower influence of antibiotic pretreatment compared to a conventional culture

Detection of some resistance markers
Turnaround time (1-2 days)

Low sensitivity, especially if sample is taken after starting antibiotic treatment

Sometimes difficult to differentiate infection from colonisation

Cost is a concern

Only detects one pathogen

Does not determine susceptibility to all antibiotics-a conventional antibiogram remains the "gold standard"

Cost is the main current concern

Not all pathogens causing respiratory tract infections are included in the multiplex panel

Does not determine susceptibility to all antibiotics-a conventional antibiogram remains the "gold standard"

MRSA: methicillin-resistant Staphylococcus aureus. 
healthcare-associated pneumonia, ventilator-associated pneumonia, or severe CAP. This study showed that applying this diagnostic method resulted in a three times higher identification of organisms in blood than using standard cultures and, more importantly, changed the antibiotic treatment in $57 \%$ of the included patients.

\section{The lung microbiome}

In recent years, new culture-independent methods have shown that the lungs, previously thought to be sterile, have a diversity of microbial communities [111,113]. These communities exist in the absence of infection and they are modified in the event of an acute or exacerbated infection [111]. The pathogenic micro-organisms found in pneumonia (e.g. S. pneumoniae) are only some of those that have access to the lower respiratory tract but can also be identified in the lung microbiota of patients without acute pneumonia [114].

CHEN et al. [114] studied the microbiome in sputum samples from 45 patients with CAP. They found similarities with healthy controls at phyla level, and at genera level they found stability for Streptococcus spp. and Neisseria spp. but changes in Moraxella spp. and Rothia spp. They concluded that Rothia spp. may be an endogenous pneumonia-causing pathogen. In elderly patients with pneumonia, Wouter et al. [115] observed three microbiota profiles strongly associated with pneumonia: lactobacilli, S. pneumoniae and Rothia spp. They suggested that pneumonia in elderly patients with dysbiosis of the upper respiratory tract microbiome had bacterial overgrowth of a single species and distinct anaerobic bacteria.

Abeles et al. [116] studied the effect of the two most commonly prescribed antibiotics in the USA (amoxicillin and azithromycin) to discern whether short-term antibiotic use had any prolonged effect on human microbiota. As antibiotics are often absorbed across the gastrointestinal tract and distributed to the tissues via the bloodstream, they expected that each would affect the microflora of each body surface tested (gut, skin and mouth). The authors found that as few as 3 days of treatment with the most commonly prescribed antibiotics could result in sustained reduction in microbiota diversity, which may have implications for the maintenance of human health and resilience to disease.

Although there is still a long way to go, we are sure that, in the coming years, better knowledge and understanding of the lung (and gut) microbiome will change our view of the physiopathology, treatment and prevention of pneumonia.

\section{Molecular techniques in clinical practice}

Rapid diagnostic tests identify a specific pathogen or help to distinguish between bacterial and viral infection; provide information about antibiotic susceptibility; monitor response to antibiotic therapy; assess prognosis; aid antimicrobial stewardship; and give information for disease surveillance.

These technologies can be applied to different patient populations ranging from outpatients (allowing safe discharge) to patients that need ICU treatment. The quick results (1-2 h) may be useful in the decision management of critical patients especially in the prompt initiation of appropriate antimicrobial therapy, a factor associated with mortality. Also, rapid identification of antibiotic resistant pathogens is central to timely isolation of patients [19]. However, there are still two major challenges to face. 1) The differentiation between colonisation and infection is still an issue for clinicians, except probably in the case of $S$. pneumoniae and we do not have enough evidence for all these techniques to draw conclusions regarding this. 2) The relative costs and outcomes of diagnostic testing must be considered when the decision to implement these rapid tests is taken by healthcare systems-further studies on cost-effectiveness are needed.

\section{Conclusions and implementation}

The combination of new and old techniques will improve our ability to detect the microbes that cause pneumonia more precisely and rapidly. This strategy will ensure that patients receive the most appropriate antimicrobial therapy and may reduce the use of broad-spectrum antibiotics, thereby reducing antibiotic resistance (figure 1). Since the majority of studies described in this review show the potential advantages of molecular techniques, such as improved sensitivity and improved speed in establishing a microbiological diagnosis, more studies are needed to systematically and rigorously evaluate their performance characteristics, determine how these new technologies will improve diagnostic testing for respiratory pathogens and effect patient management during their future implementation in daily practice. To distinguish between colonisation and infection, the use of a quantitative cut-off for the number of colony-forming units $\left(\geqslant 10^{-5}\right.$ $\mathrm{CFU} \cdot \mathrm{mL}^{-1}$, as described in previous studies) is recommended [43, 58, 62, 63]. For implementation of these techniques it is necessary to discuss with the microbiology department and the treating specialist which molecular tests need to be used and which pathogens should be targeted. Although recent publications regarding the apparent utility of multiplex molecular procedures in the detection of CAP pathogens are encouraging, significant concerns remain $[58,62,63]$. A protocol addressing these issues should be developed and evaluated. A key question in this evaluation is whether our current clinical practice needs to change. 
Will there be a change in prescribing antibiotics and, if so, would it improve antibiotic stewardship? Furthermore, a close evaluation of costs needs to be implemented. Finally, the concepts regarding the lung microbiome are very promising and are currently the focus of many studies. We believe that these concepts will change our understanding of pulmonary infection, how to prevent it and how to improve its treatment.

\section{References}

1 Centers for Disease Control and Prevention (CDC). Control of Infectious Diseases. MMWR Morb Mortal Wkly Rep 1999; 48: 621-629.

2 Naghavi M, Wang H, Lozano, R, et al. Global, regional, and national age-sex specific all-cause and cause-specific mortality for 240 causes of death, 1990-2013: a systematic analysis for the Global Burden of Disease Study 2013. Lancet 2015; 385: 117-171.

3 Musher DM, Thorner AR. Community-acquired pneumonia. N Engl J Med 2014; 371: 1619-1628.

4 Bonten MJ, Huijts SM, Bolkenbaas M, et al. Polysaccharide conjugate vaccine against pneumococcal pneumonia in adults. $N$ Engl J Med 2015; 372: 1114-1125.

5 Welte T, Torres A, Nathwani D. Clinical and economic burden of community-acquired pneumonia among adults in Europe. Thorax 2012; 67: 71-79.

6 File TM Jr, Marrie TJ. Burden of community-acquired pneumonia in North American adults. Postgrad Med 2010; 122: 130-141.

7 Song JH, Thamlikitkul V, Hsueh PR. Clinical and economic burden of community-acquired pneumonia amongst adults in the Asia-Pacific region. Int J Antimicrob Agents 2011; 38: 108-117.

8 Ewig S, Torres A. Community-acquired pneumonia as an emergency: time for an aggressive intervention to lower mortality. Eur Respir J 2011; 38: 253-260.

9 Garau J, Baquero F, Perez-Trallero E, et al. Factors impacting on length of stay and mortality of community-acquired pneumonia. Clin Microbiol Infect 2008; 14: 322-329.

10 Barlow G, Nathwani D, Williams F, et al. Reducing door-to-antibiotic time in community-acquired pneumonia: controlled before-and-after evaluation and cost-effectiveness analysis. Thorax 2007; 62: 67-74.

11 Yu KT, Wyer PC. Evidence-based emergency medicine/critically appraised topic. Evidence behind the 4-hour rule for initiation of antibiotic therapy in community-acquired pneumonia. Ann Emerg Med 2008; 51: 651-662.

12 Mandell LA, Wunderink RG, Anzueto A, et al. Infectious Diseases Society of America/American Thoracic Society consensus guidelines on the management of community-acquired pneumonia in adults. Clin Infect Dis 2007; 44: Suppl. 2, S27-S72.

13 Aliberti S, Di Pasquale M, Zanaboni AM, et al. Stratifying risk factors for multidrug-resistant pathogens in hospitalized patients coming from the community with pneumonia. Clin Infect Dis. 2012; 54: 470-478.

14 Shorr AF, Zilberberg MD, Micek ST, et al. Prediction of infection due to antibiotic-resistant bacteria by select risk factors for health care-associated pneumonia. Arch Intern Med 2008; 168: 2205-2210.

15 Prina E, Ranzani OT, Polverino E, et al. Risk factors associated with potentially antibiotic-resistant pathogens in community-acquired pneumonia. Ann Am Thorac Soc 2015; 12: 153-160.

16 Houck PM, Bratzler DW, Nsa W, et al. Timing of antibiotic administration and outcomes for Medicare patients hospitalized with community-acquired pneumonia. Arch Intern Med 2004; 164: 637-644.

17 Waterer GW, Kessler LA, Wunderink RG. Delayed administration of antibiotics and atypical presentation in community-acquired pneumonia. Chest 2006; 130: 11-15.

18 Cilloniz C, Gabarrus A, Ferrer M, et al. Community-acquired pneumonia due to multidrug and non-multidrug resistant Pseudomonas aeruginosa. Chest 2016; 150: 415-425.

19 Douglas IS. New diagnostic methods for pneumonia in the ICU. Curr Opin Infect Dis 2016; 29: 197-204.

20 Shindo $\mathrm{Y}$, Ito R, Kobayashi $\mathrm{D}$, et al. Risk factors for drug-resistant pathogens in community-acquired and healthcare-associated pneumonia. Am J Respir Crit Care Med 2013; 188: 985-995.

21 Chalmers JD, Taylor JK, Singanayagam A, et al. Epidemiology, antibiotic therapy, and clinical outcomes in health care-associated pneumonia: a UK cohort study. Clin Infect Dis 2011; 53: 107-113.

22 Van Boeckel TP, Gandra S, Ashok A, et al. Global antibiotic consumption 2000 to 2010: an analysis of national pharmaceutical sales data. Lancet Infect Dis 2014; 14: 742-750.

23 Aliberti S, Cilloniz C, Chalmers JD, et al. Multidrug-resistant pathogens in hospitalised patients coming from the community with pneumonia: a European perspective. Thorax 2013; 68: 997-999.

24 Giamarellou H, Poulakou G. Multidrug-resistant Gram-negative infections: what are the treatment options? Drugs 2009; 69: 1879-1901.

25 The Center for Disease Dynamics, Economics and Policy. The State of the World's Antibiotics 2015. Washington, The Center for Disease Dynamics, Economics and Policy (CDDEP), 2015. Available from: https:// cddep.org/sites/default/files/swa_2015_final.pdf.

26 Chahwakilian P, Huttner B, Schlemmer B, et al. Impact of the French campaign to reduce inappropriate ambulatory antibiotic use on the prescription and consultation rates for respiratory tract infections. J Antimicrob Chemother 2011; 66: 2872-2879.

27 McDonagh $\mathrm{M}$, Peterson $\mathrm{K}$, Winthrop $\mathrm{K}$, et al. Improving antibiotic prescribing for uncomplicated acute respiratory tract infections [Internet]. AHRQ Comparative Effectiveness Reviews 2016; 163: 5(16)-EHC033-EF.

28 National Action Plan for Combating Antibiotic-Resistant Bacteria. Washington, The White House, 2015. Available from: www.whitehouse.gov/sites/default/files/docs/national_action_plan_for_combating_antibotic-resistant_ bacteria.pdf.

29 U.S. Food \& Drug Administration (FDA). News Release: New FDA task force will support innovation in antibacterial drug development. Silver Spring, MD, U.S. Food \& Drug Administration (FDA), 24 September 2012. Available from: http://www.fda.gov/NewsEvents/Newsroom/PressAnnouncements/ucm320643.htm; Page last updated: September 24, 2012.

30 van der Eerden MM, Vlaspolder F, de Graaff CS, et al. Value of intensive diagnostic microbiological investigation in low- and high-risk patients with community-acquired pneumonia. Eur J Clin Microbiol Infect Dis 2005; 24: 241-249. 

community-acquired pneumonia in patients hospitalized on the basis of conventional admission criteria. Clin Infect Dis 2001; 33: 158-165.

32 Lim WS, Macfarlane JT, Boswell TC, et al. Study of community acquired pneumonia aetiology (SCAPA) in adults admitted to hospital: implications for management guidelines. Thorax 2001; 56: 296-301.

33 Waterer GW, Wunderink RG. The influence of the severity of community-acquired pneumonia on the usefulness of blood cultures. Respir Med 2001; 95: 78-82.

34 Polverino E, Torres A. Community-acquired penumonia. Minerva Anestesiol 2011; 77: 196-211.

35 Roson B, Carratala J, Verdaguer R, et al. Prospective study of the usefulness of sputum Gram stain in the initial approach to community-acquired pneumonia requiring hospitalization. Clin Infect Dis 2000; 31: 869-874.

36 Anevlavis S, Petroglou N, Tzavaras A, et al. A prospective study of the diagnostic utility of sputum Gram stain in pneumonia. J Infect 2009; 59: 83-89.

37 Johansson N, Kalin M, Giske CG, et al. Quantitative detection of Streptococcus pneumoniae from sputum samples with real-time quantitative polymerase chain reaction for etiologic diagnosis of community-acquired pneumonia. Diagn Microbiol Infect Dis 2008; 60: 255-261.

38 Resti M, Micheli A, Moriondo M, et al. Comparison of the effect of antibiotic treatment on the possibility of diagnosing invasive pneumococcal disease by culture or molecular methods: a prospective, observational study of children and adolescents with proven pneumococcal infection. Clin Ther 2009; 31: 1266-1273.

39 Song JY, Eun BW, Nahm MH. Diagnosis of pneumococcal pneumonia: current pitfalls and the way forward. Infect Chemother 2013; 45: 351-366.

40 Murdoch DR, Anderson TP, Beynon KA, et al. Evaluation of a PCR assay for detection of Streptococcus pneumoniae in respiratory and nonrespiratory samples from adults with community-acquired pneumonia. $J$ Clin Microbiol 2003; 41: 63-66.

41 Carvalho MG, Tondella ML, McCaustland K, et al. Evaluation and improvement of real-time PCR assays targeting lytA, ply, and psaA genes for detection of pneumococcal DNA. J Clin Microbiol 2007; 45: 2460-2466.

42 Tuite N, Reddington K, Barry T, et al. Rapid nucleic acid diagnostics for the detection of antimicrobial resistance in Gram-negative bacteria: is it time for a paradigm shift? J Antimicrob Chemother. 2014; 69: 1729-1733.

43 Gadsby NJ, Russell CD, McHugh MP, et al. Comprehensive molecular testing for respiratory pathogens in community-acquired pneumonia. Clin Infect Dis 2016; 62: 817-823.

44 Wellinghausen N, Siegel D, Gebert S, et al. Rapid detection of Staphylococcus aureus bacteremia and methicillin resistance by real-time PCR in whole blood samples. Eur J Clin Microbiol Infect Dis 2009; 28: 1001-1005.

45 de Paz HD, Brotons P, Munoz-Almagro C. Molecular isothermal techniques for combating infectious diseases: towards low-cost point-of-care diagnostics. Expert Rev Mol Diagn 2014; 14: 827-843.

Zhang W, Chen C, Cui J, et al. Application of loop-mediated isothermal amplification (LAMP) assay for the rapid diagnosis of pathogenic bacteria in clinical sputum specimens of acute exacerbation of COPD (AECOPD). Int J Clin Exp Med 2015; 8: 7881-7889.

47 Koncan R, Parisato M, Sakarikou C, et al. Direct identification of major Gram-negative pathogens in respiratory specimens by respiFISH ${ }^{\circledast}$ HAP Gram (-) Panel, a beacon-based FISH methodology. Eur J Clin Microbiol Infect Dis 2015; 34: 2097-2102.

48 Mok JH, Eom JS, Jo EJ, et al. Clinical utility of rapid pathogen identification using matrix-assisted laser desorption/ionization time-of-flight mass spectrometry in ventilated patients with pneumonia: a pilot study. Respirology 2016; 21: 321-328.

49 Zimmerman RK, Rinaldo CR, Nowalk MP, et al. Viral infections in outpatients with medically attended acute respiratory illness during the 2012-2013 influenza season. BMC Infect Dis 2015; 15: 87.

50 Babady NE. The FilmArray respiratory panel: an automated, broadly multiplexed molecular test for the rapid and accurate detection of respiratory pathogens. Expert Rev Mol Diagn 2013; 13: 779-788.

51 Loeffelholz MJ, Pong DL, Pyles RB, et al. Comparison of the FilmArray Respiratory Panel and Prodesse real-time PCR assays for detection of respiratory pathogens. J Clin Microbiol 2011; 49: 4083-4088.

52 Cvitkovic SV, Beovic B, Pokorn M, et al. Improvement of pneumococcal pneumonia diagnostics by the use of rt-PCR on plasma and respiratory samples. Scand J Infect Dis 2013; 45: 731-737.

53 Stralin K, Backman A, Holmberg H, et al. Design of a multiplex PCR for Streptococcus pneumoniae, Haemophilus influenzae, Mycoplasma pneumoniae and Chlamydophila pneumoniae to be used on sputum samples. APMIS 2005; 113: 99-111.

54 Stralin K, Herrmann B, Abdeldaim G, et al. Comparison of sputum and nasopharyngeal aspirate samples and of the PCR gene targets lytA and Spn9802 for quantitative PCR for rapid detection of pneumococcal pneumonia. J Clin Microbiol 2014; 52: 83-89.

55 Albrich WC, Madhi SA, Adrian PV, et al. Genomic load from sputum samples and nasopharyngeal swabs for diagnosis of pneumococcal pneumonia in HIV-infected adults. J Clin Microbiol 2014; 52: 4224-4229.

56 Stralin K, Tornqvist E, Kaltoft MS, et al. Etiologic diagnosis of adult bacterial pneumonia by culture and PCR applied to respiratory tract samples. J Clin Microbiol 2006; 44: 643-645.

57 Lorente ML, Falguera M, Nogues A, et al. Diagnosis of pneumococcal pneumonia by polymerase chain reaction (PCR) in whole blood: a prospective clinical study. Thorax 2000; 55: 133-137.

58 Abdeldaim G, Herrmann B, Molling P, et al. Usefulness of real-time PCR for lytA, ply, and Spn9802 on plasma samples for the diagnosis of pneumococcal pneumonia. Clin Microbiol Infect 2010; 16: 1135-1141.

59 Peters RP, de Boer RF, Schuurman T, et al. Streptococcus pneumoniae DNA load in blood as a marker of infection in patients with community-acquired pneumonia. J Clin Microbiol 2009; 47: 3308-3312.

60 Avni T, Mansur N, Leibovici L, et al. PCR using blood for diagnosis of invasive pneumococcal disease: systematic review and meta-analysis. J Clin Microbiol 2010; 48: 489-496.

61 Resti M, Moriondo M, Cortimiglia M, et al. Community-acquired bacteremic pneumococcal pneumonia in children: diagnosis and serotyping by real-time polymerase chain reaction using blood samples. Clin Infect Dis 2010; 51: 1042-1049.

62 Albrich WC, Madhi SA, Adrian PV, et al. Use of a rapid test of pneumococcal colonization density to diagnose pneumococcal pneumonia. Clin Infect Dis 2012; 54: 601-609. 
63 Abdeldaim GM, Stralin K, Olcen P, et al. Toward a quantitative DNA-based definition of pneumococcal pneumonia: a comparison of Streptococcus pneumoniae target genes, with special reference to the Spn9802 fragment. Diagn Microbiol Infect Dis 2008; 60: 143-150.

64 Rello J, Lisboa T, Lujan M, et al. Severity of pneumococcal pneumonia associated with genomic bacterial load. Chest 2009; 136: 832-840.

65 Cremers AJ, Hagen F, Hermans PW, et al. Diagnostic value of serum pneumococcal DNA load during invasive pneumococcal infections. Eur J Clin Microbiol Infect Dis 2014; 33: 1119-1124.

66 Elberse K, van MS, Cremers AJ, et al. Detection and serotyping of pneumococci in community acquired pneumonia patients without culture using blood and urine samples. BMC Infect Dis 2015; 15: 56.

67 Morozumi M, Chiba N, Okada T, et al. Antibiotic susceptibility in relation to genotype of Streptococcus pneumoniae, Haemophilus influenzae, and Mycoplasma pneumoniae responsible for community-acquired pneumonia in children. J Infect Chemother 2013; 19: 432-440.

68 Jain S, Self WH, Wunderink RG, et al. Community-acquired pneumonia requiring hospitalization among US Adults. N Engl J Med 2015; 373: 415-427.

69 Lui G, Ip M, Lee N, et al. Role of 'atypical pathogens' among adult hospitalized patients with communityacquired pneumonia. Respirology 2009; 14: 1098-1105.

70 Arnold FW, Summersgill JT, Lajoie AS, et al. A worldwide perspective of atypical pathogens in communityacquired pneumonia. Am J Respir Crit Care Med 2007; 175: 1086-1093.

71 Cilloniz C, Torres A, Niederman M, et al. Community-acquired pneumonia related to intracellular pathogens. Intensive Care Med 2016; 42: 1374-1386.

72 Karhu J, la-Kokko TI, Vuorinen T, et al. Lower respiratory tract virus findings in mechanically ventilated patients with severe community-acquired pneumonia. Clin Infect Dis 2014; 59: 62-70.

73 Lee N, Ison MG. Diagnosis, management and outcomes of adults hospitalized with influenza. Antivir Ther (Lond) 2012; 17: 143-157.

74 Harper SA, Bradley JS, Englund JA, et al. Seasonal influenza in adults and children-diagnosis, treatment, chemoprophylaxis, and institutional outbreak management: clinical practice guidelines of the Infectious Diseases Society of America. Clin Infect Dis 2009; 48: 1003-1032.

75 Chan MC, Lee N, Ngai KL, et al. Clinical and virologic factors associated with reduced sensitivity of rapid influenza diagnostic tests in hospitalized elderly patients and young children. J Clin Microbiol 2014; 52: 497-501.

76 Lee N, Leo YS, Cao B, et al. Neuraminidase inhibitors, superinfection and corticosteroids affect survival of influenza patients. Eur Respir J 2015; 45: 1642-1652.

77 Brendish NJ, Schiff HF, Clark TW. Point-of-care testing for respiratory viruses in adults: the current landscape and future potential. $J$ Infect 2015; 71: 501-510.

78 Bautista E, Chotpitayasunondh T, Gao Z, et al. Clinical aspects of pandemic 2009 influenza A (H1N1) virus infection. N Engl J Med 2010; 362: 1708-1719.

79 Chartrand C, Leeflang MM, Minion J, et al. Accuracy of rapid influenza diagnostic tests: a meta-analysis. Ann Intern Med 2012; 156: 500-511.

80 Centers for Disease Control and Prevention (CDC). Clinical Description \& Lab Diagnosis of Influenza, 2015. http://www.cdc.gov/flu/professionals/diagnosis/. Date last reviewed: 27 October, 2015. Date last updated: 16 December, 2015

81 Hurt AC, Chotpitayasunondh T, Cox NJ, et al. Antiviral resistance during the 2009 influenza A H1N1 pandemic: public health, laboratory, and clinical perspectives. Lancet Infect Dis 2012; 12: 240-248.

82 Lee N, Chan PK, Hui DS, et al. Viral loads and duration of viral shedding in adult patients hospitalized with influenza. J Infect Dis 2009; 200: 492-500.

83 Li TC, Chan MC, Lee N. Clinical implications of antiviral resistance in influenza. Viruses 2015; 7: 4929-4944.

84 Lee WY, Mokhlesi B. Diagnosis and management of obesity hypoventilation syndrome in the ICU. Crit Care Clin 2008; 24: 533-549.

85 Yeh E, Luo RF, Dyner L, et al. Preferential lower respiratory tract infection in swine-origin 2009 A(H1N1) influenza. Clin Infect Dis 2010; 50: 391-394.

86 Branche AR, Walsh EE, Formica MA, et al. Detection of respiratory viruses in sputum from adults by use of automated multiplex PCR. J Clin Microbiol 2014; 52: 3590-3596.

87 Walsh EE, Peterson DR, Kalkanoglu AE, et al. Viral shedding and immune responses to respiratory syncytial virus infection in older adults. J Infect Dis 2013; 207: 1424-1432.

88 Lee N, Qureshi ST. Other viral pneumonias: coronavirus, respiratory syncytial virus, adenovirus, hantavirus. Crit Care Clin 2013; 29: 1045-1068.

89 Lee N, Lui GC, Wong KT, et al. High morbidity and mortality in adults hospitalized for respiratory syncytial virus infections. Clin Infect Dis 2013; 57: 1069-1077.

90 Zumla A, Al-Tawfiq JA, Enne VI, et al. Rapid point of care diagnostic tests for viral and bacterial respiratory tract infections--needs, advances, and future prospects. Lancet Infect Dis 2014; 14: 1123-1135.

91 Scanvic A, Courdavault L, Sollet JP, et al. Interet de la PCR temps reel Cepheid Xpert SAMR/SA sur GeneXpert ${ }^{\circ}$ DX System dans la prise en charge de la bacteriemie a staphylocoque [Interest of real-time PCR Xpert MRSA/SA on GeneXpert ${ }^{\circ}$ DX System in the investigation of staphylococcal bacteremia]. Pathol Biol (Paris) 2011; 59: 67-72. Prachayangprecha S, Schapendonk CM, Koopmans MP, et al. Exploring the potential of next-generation sequencing in detection of respiratory viruses. J Clin Microbiol 2014; 52: 3722-3730.

93 Phin N, Parry-Ford F, Harrison T, et al. Epidemiology and clinical management of Legionnaires' disease. Lancet Infect Dis 2014; 14: 1011-1021.

94 Lee N, Chan MC, Lui GC, et al. High viral load and respiratory failure in adults hospitalized for respiratory syncytial virus infections. J Infect Dis 2015; 212: 1237-1240.

95 Meyer Sauteur PM, van Rossum AM, Vink C. Mycoplasma pneumoniae in children: carriage, pathogenesis, and antibiotic resistance. Curr Opin Infect Dis 2014; 27: 220-227.

96 Iregui M, Ward S, Sherman G, et al. Clinical importance of delays in the initiation of appropriate antibiotic treatment for ventilator-associated pneumonia. Chest 2002; 122: 262-268.

97 Kumar A, Ellis $\mathrm{P}$, Arabi Y, et al. Initiation of inappropriate antimicrobial therapy results in a fivefold reduction of survival in human septic shock. Chest 2009; 136: 1237-1248. 
Thurman KA, Walter ND, Schwartz SB, et al. Comparison of laboratory diagnostic procedures for detection of Mycoplasma pneumoniae in community outbreaks. Clin Infect Dis 2009; 48: 1244-1249.

99 Shimada T, Noguchi Y, Jackson JL, et al. Systematic review and metaanalysis: urinary antigen tests for Legionellosis. Chest 2009; 136: 1576-1585.

100 Murdoch DR, Podmore RG, Anderson TP, et al. Impact of routine systematic polymerase chain reaction testing on case finding for Legionnaires' disease: a pre-post comparison study. Clin Infect Dis 2013; 57: 1275-1281.

101 Martin-Loeches I, Deja M, Koulenti D, et al. Potentially resistant microorganisms in intubated patients with hospital-acquired pneumonia: the interaction of ecology, shock and risk factors. Intensive Care Med 2013; 39: 672-681.

102 Garnacho-Montero J, Ortiz-Leyba C, Fernandez-Hinojosa E, et al. Acinetobacter baumannii ventilator-associated pneumonia: epidemiological and clinical findings. Intensive Care Med 2005; 31: 649-655.

103 Enne VI, Personne Y, Grgic L, et al. Aetiology of hospital-acquired pneumonia and trends in antimicrobial resistance. Curr Opin Pulm Med 2014; 20: 252-258.

104 Carbonnelle E, Mesquita C, Bille E, et al. MALDI-TOF mass spectrometry tools for bacterial identification in clinical microbiology laboratory. Clin Biochem 2011; 44: 104-109.

105 Sparbier K, Schubert S, Weller U, et al. Matrix-assisted laser desorption ionization-time of flight mass spectrometry-based functional assay for rapid detection of resistance against $\beta$-lactam antibiotics. J Clin Microbiol 2012; 50: 927-937.

106 Shorr AF, Chan CM, Zilberberg MD. Diagnostics and epidemiology in ventilator-associated pneumonia. Ther Adv Respir Dis 2011; 5: 121-130.

107 Falcone M, Russo A, Giannella M, et al. Individualizing risk of multidrug-resistant pathogens in communityonset pneumonia. PLoS ONE 2015; 10: e0119528.

108 Jauneikaite E, Tocheva AS, Jefferies JM, et al. Current methods for capsular typing of Streptococcus pneumoniae. J Microbiol Methods 2015; 113: 41-49.

109 Lee B, Boucher HW. Targeting antimicrobial-resistant bacterial respiratory tract pathogens: it is time to 'get smart'. Curr Opin Pulm Med 2015; 21: 293-303.

110 Schulte B, Eickmeyer H, Heininger A, et al. Detection of pneumonia associated pathogens using a prototype multiplexed pneumonia test in hospitalized patients with severe pneumonia. PLoS One 2014; 9: e110566.

111 Charlson ES, Bittinger K, Haas AR, et al. Topographical continuity of bacterial populations in the healthy human respiratory tract. Am J Respir Crit Care Med 2011; 184: 957-963.

112 Vincent JL, Brealey D, Libert N, et al. Rapid diagnosis of infection in the critically ill, a multicenter study of molecular detection in bloodstream infections, pneumonia, and sterile site infections. Crit Care Med 2015; 43: 2283-2291.

113 Dickson RP, Erb-Downward JR, Martinez FJ, et al. The microbiome and the respiratory tract. Annu Rev Physiol 2016; 78: 481-504.

114 Chen C, Shen T, Tian F, et al. New microbiota found in sputum from patients with community-acquired pneumonia. Acta Biochim Biophys Sin (Shanghai) 2013; 45: 1039-1048.

115 de Steenhuijsen Piters WA, Huijskens EG, Wyllie AL, et al. Dysbiosis of upper respiratory tract microbiota in elderly pneumonia patients. ISME J 2016; 10: 97-108.

116 Abeles SR, Jones MB, Santiago-Rodriguez TM, et al. Microbial diversity in individuals and their household contacts following typical antibiotic courses. Microbiome 2016; 4: 39. 